\title{
The Diagnostic Accuracy of Contrast-Enhanced CT of the Neck for the Investigation of Sialolithiasis
}

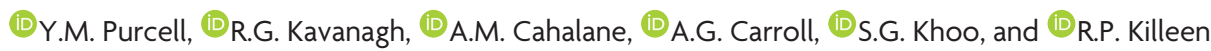

\begin{abstract}
BACKGROUND AND PURPOSE: Sialolithiasis is a common benign pathology affecting the salivary glands but it is unclear if contrastenhanced CT, which is commonly used for investigation of head and neck pathology, can identify calculi as accurately as noncontrast CT. The aim of this study was to assess the diagnostic accuracy of contrast-enhanced CT of the neck in the diagnosis of sialolithiasis compared with noncontrast CT of the neck used as the criterion standard.
\end{abstract}

MATERIALS AND METHODS: This was a retrospective, case-control study of 92 consecutive cases in 90 patients who underwent both noncontrast CT of the neck and contrast-enhanced CT of the neck in 2 tertiary referral centers from January 2011 to December 2015 for investigation of sialolithiasis. Axial 3-mm-section images were assessed by a fellowship-trained diagnostic neuroradiologist and diagnostic neuroradiology fellow in consensus. Blinded assessment of the contrast-enhanced CT of the neck was performed first, followed by noncontrast CT of the neck after a 2-week interval. The presence or absence of a stone and stone location and size were documented. Statistical analysis was undertaken to assess the agreement between CT protocols and calculate the sensitivity and specificity of contrastenhanced CT of the neck.

RESULTS: Fifty calculi were identified on noncontrast CT of the neck in 31 cases; and 48 calculi, in 31 cases on contrast-enhanced CT of the neck. No calculi were identified in the remaining 61 cases. The sensitivity and specificity of contrast-enhanced CT of the neck in the detection of sialolithiasis was $96 \%(95 \% \mathrm{Cl}, 86.3 \%-99.5 \%)$ and $100 \%(95 \% \mathrm{Cl}, 94.1 \%-100 \%)$, respectively. The positive predictive value of contrast-enhanced CT of the neck was $100 \%$ (95\% Cl, 92.6\%-100\%), and the negative predictive value was $96.8 \%$ (95\% Cl, 89\%-99.6\%). The accuracy of contrast-enhanced CT of the neck in diagnosing the presence or absence of salivary calculi was $98 \%$.

CONCLUSIONS: Contrast-enhanced CT of the neck is accurate in the detection of sialolithiasis, with no difference in diagnostic accuracy compared with noncontrast $\mathrm{CT}$ of the neck.

ABBREVIATIONS: $C E C T N=$ contrast-enhanced $C T$ of the neck; $N C C T N=$ noncontrast $C T$ of the neck

$\mathbf{S}$ alolithiasis is defined as the formation of calculi within the salivary glands. ${ }^{1}$ It is the most common non-neoplastic condition that affects them. Calculi, which are often multiple, cause partial or complete obstruction of the salivary duct, resulting in obstructive sialadenitis, which may manifest as acute or chronic unilateral gland swelling and pain. ${ }^{2-6}$ Symptoms typically arise during eating because chewing food precipitates salivation. ${ }^{7}$ Sialadenitis can be complicated by bacterial infection and abscess

Received February 16, 2017; accepted after revision June 16.

From the Departments of Radiology (Y.M.P., R.G.K., A.M.C., A.G.C., R.P.K.) and Otolaryngology (S.G.K.), St. Vincent's University Hospital, Elm Park, Dublin, Ireland; and Departments of Otolaryngology (S.G.K.) and Radiology (R.P.K.), Royal Victoria Eye and Ear Hospital, Dublin, Ireland.

Please address correspondence to Yvonne M. Purcell, MD, Radiology Department, St. Vincent's University Hospital, Elm Park, Dublin 4, Ireland; e-mail: yvonne.purcell@gmail.com

http://dx.doi.org/10.3174/ajnr.A5353 formation. ${ }^{5,8}$ While calculi in the distal submandibular duct may be readily detectable clinically by bimanual palpation, calculi in the proximal submandibular duct or gland and parotid duct or gland depend on imaging for diagnosis. ${ }^{8}$

Noncontrast CT of the neck (NCCTN) has been recommended for the evaluation of salivary calculi because it is sensitive for the detection of calcifications. ${ }^{9}$ One of the drawbacks of NCCTN is that it provides less detail of duct dilation and intraductal or glandular pathology than contrast-enhanced CT of the neck (CECTN). ${ }^{10}$ Traditionally, the use of CECTN in this setting has been in addition to the NCCTN rather than as a stand-alone test. ${ }^{11}$ Concerns have been raised that on CECTN, blood vessels may simulate small sialoliths and lead to a false-positive diagnosis or reduce diagnostic certainty. ${ }^{9,11}$ CECTN has been used for the evaluation of complicated stone disease in a suspected abscess or an inflammatory process after calculi have been identified on 


\begin{tabular}{|c|c|c|c|c|}
\hline Demographics $(n=92)$ & All $(n=92)$ & Stone $(n=31)$ & $\begin{array}{c}\text { No Stone } \\
(n=61)\end{array}$ & $\begin{array}{c}P \\
\text { Value }\end{array}$ \\
\hline Mean age (range) (yr) & $50(20-85)$ & $50(20-79)$ & $49(20-85)$ & .76 \\
\hline $\operatorname{Sex}(M / F)(\%)$ & $43: 57$ & $48: 52$ & $41: 59$ & .52 \\
\hline \multicolumn{5}{|l|}{ Referral source (\%) } \\
\hline $\mathrm{H} \& \mathrm{~N}$ & $79.3 \%(73 / 92)$ & $83.9 \%(26 / 31)$ & $77 \%(47 / 61)$ & \\
\hline ED & $15.2 \%(14 / 92)$ & $12.9 \%(4 / 31)$ & $16.4 \%(10 / 61)$ & \\
\hline Other & $5.4 \%(5 / 92)$ & $3.2 \%(1 / 31)$ & $6.6 \%(4 / 61)$ & \\
\hline \multicolumn{5}{|l|}{ Stone location $(n=50)$} \\
\hline Submandibular system & & $70 \%(35 / 50)$ & & \\
\hline Gland & & $38 \%(19 / 50)$ & & \\
\hline Duct & & $32 \%(16 / 50)$ & & \\
\hline Parotid system & & $30 \%(15 / 50)$ & & \\
\hline Gland & & $24 \%(12 / 50)$ & & \\
\hline Duct & & $6 \%(3 / 50)$ & & \\
\hline
\end{tabular}

Note:- H\&N indicates head and neck; ED, emergency department.

NCCTN. ${ }^{9,11,12}$ The use of NCCTN alone gives less detail of glandular and periglandular inflammation. ${ }^{9}$ The addition of CECTN, however, will cause an increase in the patient radiation dose.

The purpose of this study was to determine the accuracy of CECTN alone in the detection of salivary calculi compared with NCCTN alone. A secondary objective was to calculate the patient radiation dose when performing both NCCTN and CECTN.

\section{MATERIALS AND METHODS \\ Subjects}

This retrospective, case-control study was conducted at St. Vincent's University Hospital, a tertiary care, university-affiliated medical center in Dublin, Ireland. The local institutional review board approved this study and waived the requirement for written informed consent.

An electronic search was performed of all consecutive CT neck examinations performed during a 5-year period between January 1, 2011, and December 31, 2015, using the search terms "calculus/ calculi," "stone/stones," and "sialolith." Data were collected by electronic query of the hospital Radiology Information System and PACS.

The inclusion criteria were patients older than 16 years of age who had both a NCCTN and CECTN performed during a single visit for the investigation of suspected salivary stones.

\section{CT Image Acquisition}

NCCTN and CECTN were acquired on a 64-row multidetector CT scanner (Somatom Sensation 64; Siemens, Erlangen, Germany) in the craniocaudal direction with a collimation of $0.6 \mathrm{~mm}$, gantry rotation of $500 \mathrm{~ms}$, and a pitch of 0.9 . Automated dosecontrol software was used with $120-\mathrm{kV}$ (peak) and reference milliampere-second (maximum, 200). For the CECTN, $100 \mathrm{~mL}$ of nonionic, iodinated low-osmolar contrast medium (Niopam, iopamidol, $340 \mathrm{mg}$ I/mL; Bracco Imaging, Milan, Italy) was injected through a 20-ga or larger cannula, typically sited in the antecubital fossa, at a rate of $2 \mathrm{~mL} / \mathrm{s}$ with acquisition performed after a delay of 60 seconds. Images for the NCCTN and CECTN were reconstructed with a $512 \times 512$ matrix and a smooth kernel, with 3-mm axial-section-thickness images set as standard and 3-mm coronal and sagittal reformats. Images were reviewed with syngo Portal Radiologist (Siemens).

\section{Image Analysis}

The NCCTN and CECTN were reviewed by both a fellowship-trained diagnostic neuroradiologist and a diagnostic neuroradiology fellow in consensus. The reviewers were blinded to patient details and formal radiology reports. Blinded review of the CECTN was performed first. Following a 2-week interval, a blinded interpretation of the NCCTN was performed. Three-millimeter axial images were inspected, with 3-mm coronal and sagittal reconstructions used for problem-solving. The images were reviewed on soft-tissue neck (center $=$ $50 \mathrm{HU}$, width $=350 \mathrm{HU}$ ), angiographic lumen (center $=100$, width $=700)$, and bone (center $=500$, width $=1500)$ window settings to optimize detection of calculi. These 3 standard window settings were adjusted manually for every case as the reviewers saw fit. The reviewers recorded the presence or absence of stones, stone location (submandibular or parotid system, gland, or duct; anterior or posterior portion of the duct), stone size, and number of stones. The size of each stone was expressed as the maximal diameter determined on a single axial section. In addition, we recorded the following data: patient demographics (age and sex), the referral source, and the presence of salivary gland masses in the study population.

\section{Statistical Analysis}

The accuracy of CECTN in determining the frequency and location of salivary calculi was compared with that of NCCTN used as the criterion standard. Descriptive values were used for patient and stone demographics (actual values, percentages). Statistical analysis was performed by using STATA Statistical Software, Release 12 (StataCorp, College Station, Texas). The sensitivity, specificity, and likelihood ratios were calculated for CECTN in the detection of salivary calculi, with the $95 \%$ confidence interval calculated as the measure of variance. The difference in the prevalence of sialolithiasis among different referrers was calculated with the $\chi^{2}$ test of proportions.

\section{RESULTS}

Our search yielded 370 potential cases performed during the 5 -year study period. Of these, 92 sets of CT examinations in 90 patients met the inclusion criteria: age older than 16 years and both NCCTN and CECTN performed contemporaneously for the investigation of suspected salivary stones (1 patient had 3 sets of CT examinations) (Table). There were 38 male (42\%) and 52 female $(58 \%)$ patients with a mean age of 50 years (range, $20-85$ years). Fifty calculi were identified in 31 patients on NCCTN (the criterion standard), which gives a prevalence of $34 \%$. One patient had 8 calculi, 1 patient had 4 calculi, 1 patient had 3 calculi, 7 patients had 2 calculi, and 21 patients had 1 calculus. Calculi were not present in 59/90 patients (61/92 total cases) on NCCTN.

On CECTN, all patients were correctly categorized as having or not having salivary stones; 48 calculi were identified in 31 patients on CECTN compared with NCCT as the criterion standard. 


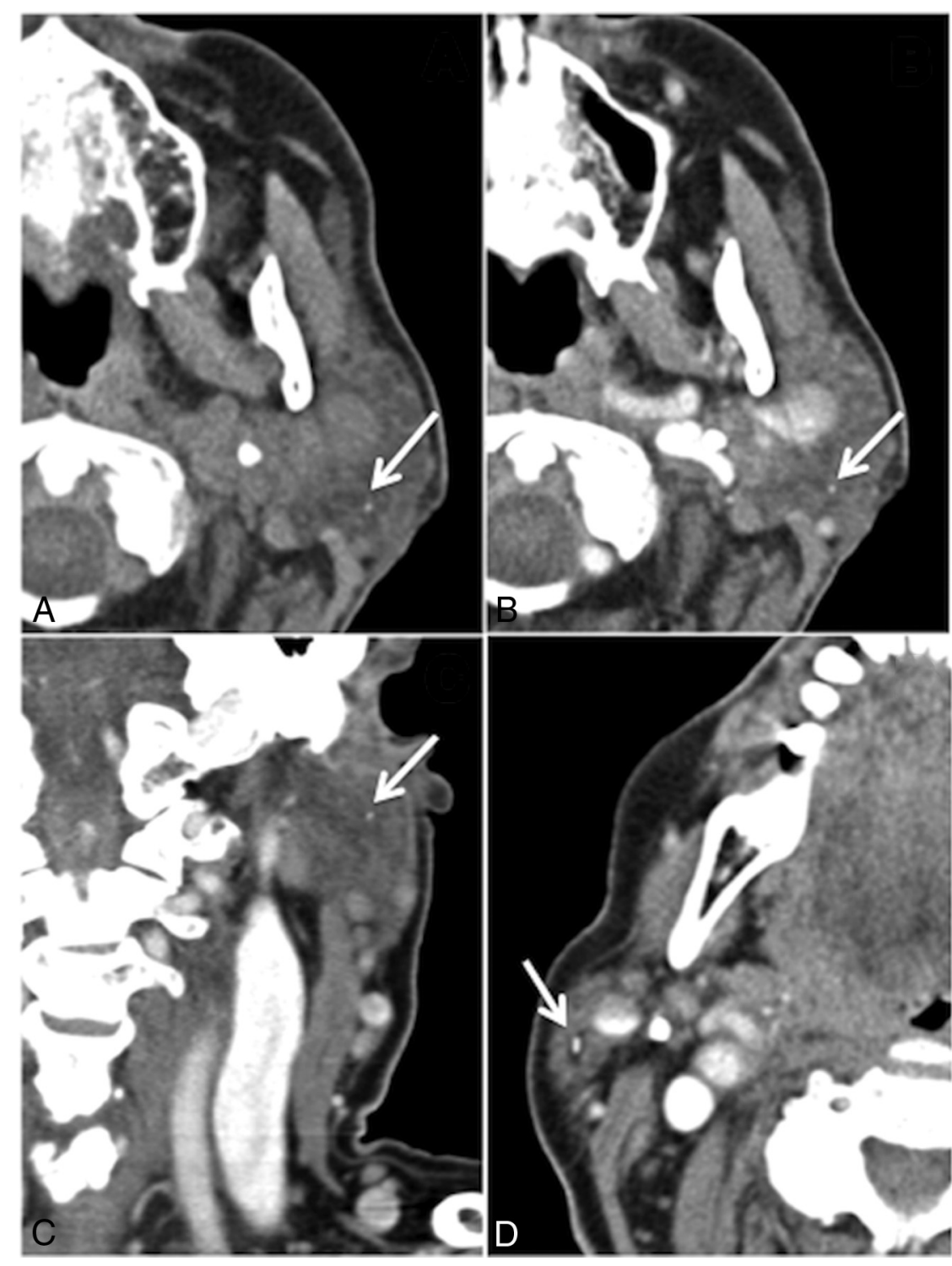

FIG 1. False-negative case. A, Noncontrast CT shows a 1-mm calculus in the superficial lobe of the left parotid gland (arrow), missed on initial review of the CECTN, though it is present on axial (B) and coronal (C, arrows) reconstructions. The distraction is an obstructing 3-mm calculus in the right parotid gland $(D$, arrow). Note the surrounding intraglandular ductal dilation.

Zero calculi were identified in the remaining 59 patients on CECTN (59/90 patients, 61/92 total cases).

The overall sensitivity of CECTN for the detection of salivary stones was 96\% (95\% CI, 86.3\%-99.5\%), and the specificity was $100 \%$ (95\% CI, 94.1\%-100\%). The positive predictive value of CECT was $100 \%$ (95\% CI, 92.6\%-100\%), and the negative predictive value was $96.8 \%$ (95\% CI, 89\%-99.6\%). The positive likelihood ratio was infinity, and the negative likelihood ratio was 0.04 (95\% CI, 0.01-0.16). The accuracy of CECTN in diagnosing the presence or absence of salivary stones in our study was $98 \%$.

Two calculi were not identified on CECTN that were identified on the criterion standard NCCTN. Both missed calculi were asymptomatic. The first missed stone was a 1-mm calculus in the left parotid gland in a patient with a symptomatic 3-mm calculus in the right parotid gland (Fig $1 A$ ). This was visible on the CECTN in hindsight but was not seen at the time of the review (Fig $1 B$ ). It was also visible on the coronal reconstruction (Fig 1C). The sec- ond missed stone was a 2-mm calculus in the left parotid gland in a patient with multiple bilateral calculi and calcified lymph nodes, which, in retrospect, was visible on the CECTN.

Of the 92 CT studies, 73 (79.3\%) referrals came from head and neck surgery; 14 (15.2\%), from the emergency department; and 5 (5.4\%), from other medical specialties (oncology, cardiology, nephrology, gastroenterology). Of the 73 referrals from head and neck surgery, 26 had calculi, which is a prevalence of $35.6 \%$. Four of the 14 emergency department referrals and 1 of the 5 referrals from other sources had calculi, giving a prevalence of $28.6 \%$ and $20 \%$, respectively. The difference in the prevalence of sialolithiasis among different referrers was not statistically significant using the $\chi^{2}$ test of proportions $(P=$ .44) (Table).

The exact locations of the 50 calculi identified are outlined in the Table. Seventy percent of the total calculi $(35 / 50)$ were in the submandibular system; $38 \%$ $(19 / 50)$, in the submandibular gland and $32 \%(16 / 50)$, in the submandibular duct. Thirty percent $(15 / 50)$ of the total calculi were in the parotid system; $24 \%$ $(12 / 50)$, in the parotid gland and $6 \%(3 /$ 50 ), in the parotid duct. The calculi ranged from $1 \mathrm{~mm}$ to $2 \mathrm{~cm}$, with a mean diameter of $6 \pm 5 \mathrm{~mm}$.

We identified 3 masses: Fine-needle aspiration was performed in 2 cases, and the third case was lost to follow-up. Cytology demonstrated a benign cyst containing inflammatory debris, and the other case was suggestive of an acute inflammatory process with inflammatory cells and degenerate stromal elements.

The mean total dose-length product of the NCCTN was 290.8 $\mathrm{mGy} \times \mathrm{cm}$, and of the CECTN, it was $366.9 \mathrm{mGy} \times \mathrm{cm}$. In patients having both CTs, the mean dose-length product was $692.7 \pm 323.5 \mathrm{mGy} \times \mathrm{cm}$. If the NCCTN had been omitted from each examination, the mean reduction in the effective dose would have been $1.6 \mathrm{mSv}$, with a standard neck conversion factor of 0.0059 , assuming the use of a head CT dose phantom of $16 \mathrm{~cm}$. This is a mean relative dose reduction of $42 \%$ per case.

\section{DISCUSSION}

Sialolithiasis is the most common benign disorder of the salivary glands. ${ }^{1}$ Eighty percent to $90 \%$ of calculi are found in the submandibular system, due to the more viscous, alkaline saliva and upward drainage of the duct promoting stasis. ${ }^{13,14}$ Parotid and sublingual calculi account for only $5 \%-10 \%$ and $0 \%-5 \%$ of cases, respectively. ${ }^{15}$ In our study, $70 \%$ of the total calculi (35/50) were 
located in the submandibular system; 38\% (19/50), in the submandibular gland and 32\% (16/50), in the submandibular duct. This figure is slightly less than rates published in the literature and may be because we only imaged symptomatic patients; those with small calculi may be asymptomatic or those with distal ductal calculi may be clinically managed without imaging. Thirty percent $(15 / 50)$ of the total calculi were in the parotid system; $24 \%$ $(12 / 50)$, in the parotid gland and 6\% (3/50), in the parotid duct. Postmortem studies have indicated that the prevalence of salivary calculi in the population is $1.2 \% .^{10,16,17}$ The peak incidence occurs from 30 to 60 years of age. ${ }^{5}$ The mean patient age in our study was 50 years, with a range of 25-85 years and a slight female preponderance of $57 \%$ (Table), correlating with that in previously published reports. ${ }^{18}$ Clinically symptomatic calculi are less fre-

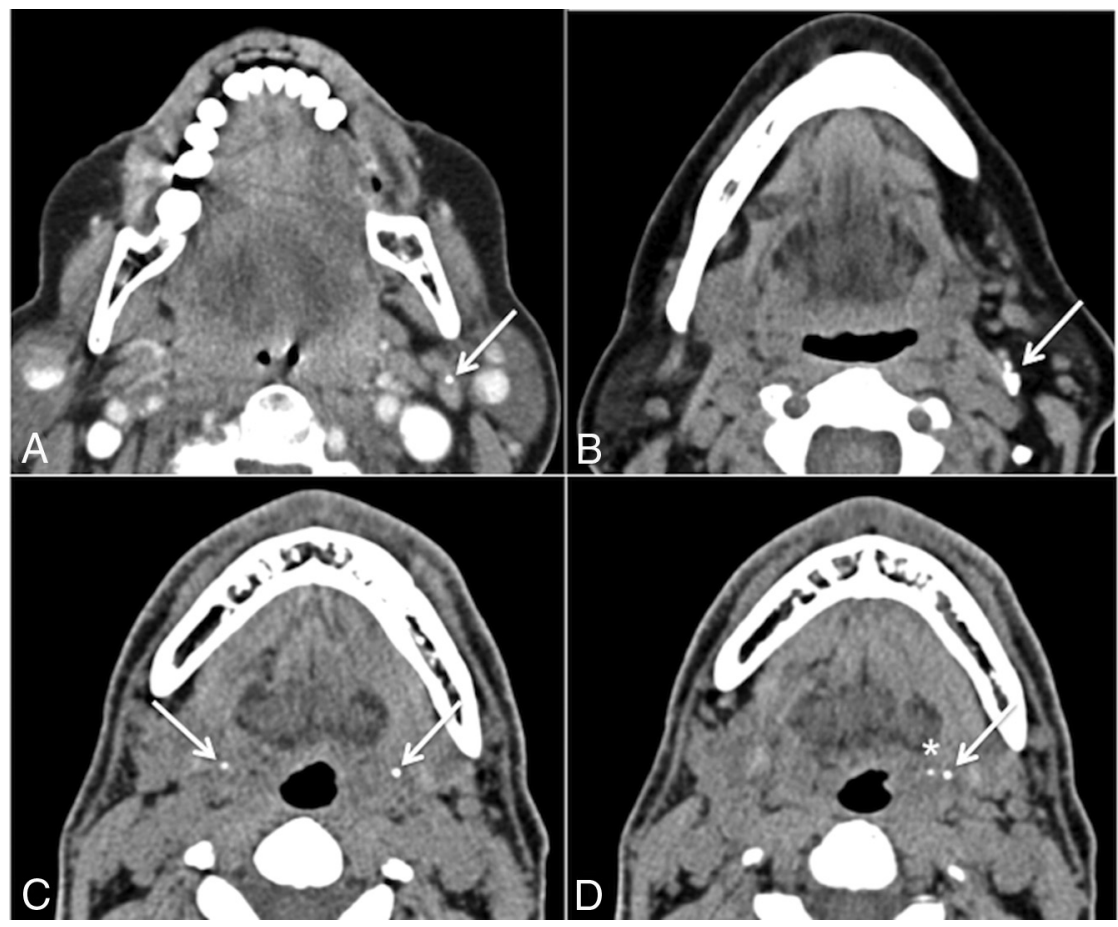

FIG 2. Potential false-positives. $A$ and $B$, Calcified lymph nodes. $C$, Bilateral calcification of the stylohyoid ligaments (arrows). D, Calcification of the left stylohyoid ligament (arrow) with an adjacent tonsillolith (asterisk) in the left palatine tonsil.
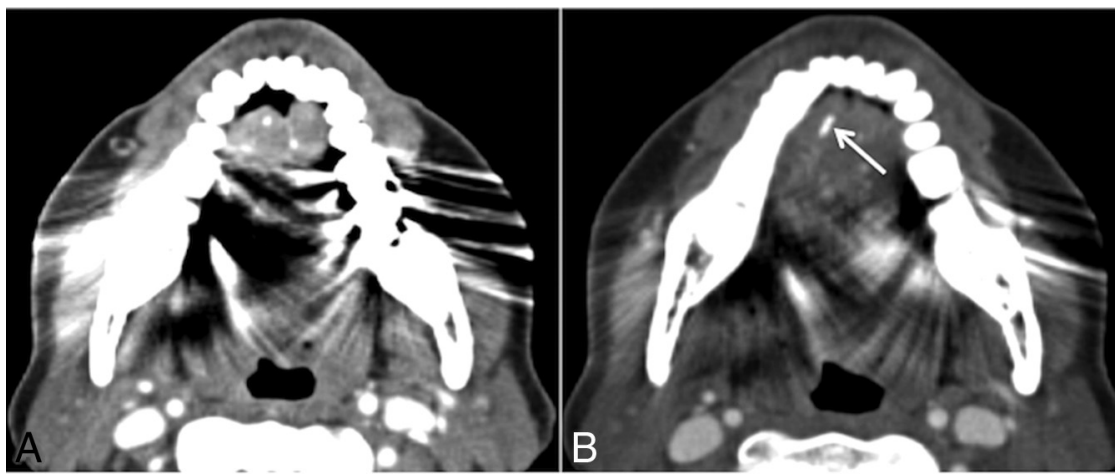

FIG 3. Potential false-negative. A, Axial contrast-enhanced CT. Significant streak artifacts in the floor of the mouth due to dental amalgam. $B$, Single section inferiorly with windows adjusted manually, revealing an obstructing calculus (arrow) in the right anterior submandibular duct. One should be cautious when analyzing the floor of the mouth because this is a classic location for obstructing submandibular duct calculi and can easily be missed. quent; an incidence of $27-59$ cases per million population per year was reported in a review of 15 health regions in England between 1991 and 1995, though a higher incidence rate than previously reported was demonstrated in a nationwide population-based study by Schrøder et al, ${ }^{18}$ of 14.10 per 100,000 person-years. ${ }^{4,19,20}$ The prevalence of salivary calculi in our study was $34 \%$. There was no significant difference in stone prevalence between patient groups referred by different specialties using the $\chi 2$ test of proportions $(P=.44)$.

Imaging techniques available for investigation of salivary calculi include conventional radiography, digital subtraction sialography, sonography, CT, and MR sialography. Conventional radiography is dependent on stone composition, and only about $20 \%$ of calculi are radiopaque. ${ }^{21}$ The sensitivity of digital subtraction sialography ranges from $96 \%$ to $100 \%$, and the specificity, from $88 \%$ to $91 \% .^{22}$ It is contraindicated in the acute setting due to the potential exacerbation of symptoms associated with infection. ${ }^{9}$ Sonography has been reported to have a sensitivity ranging from $59.1 \%$ to $93.7 \%$, and a specificity, from $86.7 \%$ to $100 \% .^{22,23}$ The sensitivity and specificity of MR imaging sialography have been reported to be $91 \%$ and $94 \%-97 \%$, respectively. ${ }^{19}$

CT of the neck with and without contrast is given a rating of 5 by the American College of Radiology, defined as "may be appropriate," in selected cases if a sialolith is suspected. ${ }^{11}$ The disadvantage of NCCTN is the lack of detail about potential complications of an obstructing calculus or an alternative diagnosis, such as a salivary tumor. ${ }^{12}$ CECTN, while useful for delineation of glandular inflammation or abscess, carries with it the risks associated with intravenous contrast such as allergy, anaphylaxis, extravasation, acute kidney injury, and so forth. Performance of both techniques results in an additional radiation dose to the patient. In our study, a mean reduction in effective dose of $1.6 \mathrm{mSv}$ or a mean relative dose reduction of $42 \%$ per case would have been achieved if NCCTN had been omitted from the protocol (using a standard neck conversion factor of 0.0059 , assuming use of a head CT dose phantom of $16 \mathrm{~cm}$ ).

Contrary to previous reports that postulated that contrast may obscure visualization of a calculus, ${ }^{9,11}$ there were no false-positives in our study; calculi can be confidently diagnosed on CECTN. This is the first study, to our knowledge, to directly compare CECTN with 
NCCTN, used as the criterion standard, for the investigation of suspected salivary calculi.

We report a sensitivity of $96 \%(95 \%$ CI, 86.3\%-99.5\%) and a specificity of $100 \%$ (95\% CI, $94.1 \%-100 \%$ ) for CECTN in the detection of salivary calculi. In this study, CECTN had an accuracy of $98 \%$ for the evaluation of salivary calculi. The diagnoses of the $66 \%$ of patients who did not have calculi included acute or chronic sialoadenitis (secondary to infective, granulomatous, or autoimmune etiology), ductal strictures, or cervical lymphadenopathy. We identified 3 masses: Fine-needle aspiration was performed in 2 cases, both benign, and the third case was lost to follow-up.

This study raises the question of the utility of the NCCTN before the CECTN if both studies are being performed, given the accuracy of CECTN in the diagnosis of salivary calculi. The introduction of recent technologic advances such as dual-energy CT may obviate performing both CT examinations. Dual-energy CT acquires images at 2 different energy levels simultaneously and uses the attenuation differences at the different energy levels for deriving additional information. Virtual unenhanced images can be generated from each dataset of a postcontrast CT by extracting the attenuation component attributed to the presence of iodine, thereby removing the need for a separate noncontrast CT and, hence, reducing the dose. ${ }^{24,25}$

There were no false-positives in our study; all calculi correctly identified on the CECTN were identified on the NCCTN. During this review, we encountered other calcifications in the region of the salivary glands that could be erroneously labeled sialoliths. Entities that radiologists should be aware of that could potentially hamper CT interpretation include calcified lymph nodes (Fig $2 \mathrm{~A}$, $-B)$; calcification of the stylohyoid ligament, which can be bilateral (Fig 2C); tonsilloliths (Fig 2D); and phleboliths. Potential falsenegatives may be caused by dental amalgam causing metal streak artifacts in the floor of the mouth obscuring a calculus, particularly in the region of the anterior submandibular duct (Fig 3). This is a common location of symptomatic stones that can be easily overlooked if one is not cautious. Radiologists should maximize the basic tools at their disposal, namely manual adjustment of all 3 aforementioned standard window settings. Metal streak artifacts are common and caused by multiple mechanisms (beamhardening, scatter, noise, undersampling, motion, and conebeam or windmill effects). ${ }^{26}$ Manufacturers offer an array of techniques to reduce metal streak artifacts, such as filtered back-projection, linear interpolation, selective Algebraic Reconstruction Technique, and the Metal Deletion Technique. ${ }^{27}$ These techniques were not routinely available at our institution during the study period.

There were 2 false-negatives in our study as previously mentioned; both were asymptomatic intraglandular calculi within the parotid gland.

There are several limitations to this study. It was retrospective and based on radiologic findings only. There was no surgical or pathologic correlation. The clinical outcome of the patients included is not known; therefore, we did not establish which calculi were clinically relevant.

\section{CONCLUSIONS}

Contrast-enhanced CT of the neck is accurate in the diagnosis of salivary calculi. If single-phase CECTN instead of dual-phase CT (NCCTN and CECTN) is performed, there is a potential to reduce the effective radiation dose to the patient.

\section{REFERENCES}

1. Kiringoda R, Eisele DW, Chang JL. A comparison of parotid imaging characteristics and sialendoscopic findings in obstructive salivary disorders. Laryngoscope 2014;124:2696-701 CrossRef Medline

2. Marchal F, Dulguerov P, Becker M, et al. Specificity of parotid sialendoscopy. Laryngoscope 2001;111:264-71 CrossRef Medline

3. Sigismund PE, Zenk J, Koch M, et al. Nearly 3,000 salivary stones: some clinical and epidemiologic aspects. Laryngoscope 2015;125: 1879-82 CrossRef Medline

4. Escudier MP, McGurk M. Symptomatic sialoadenitis and sialolithiasis in the English population: an estimate of the cost of hospital treatment. Br Dent J 1999;186:463-66 Medline

5. Lustmann J, Regev E, Melamed Y. Sialolithiasi: a survey on 245 patients and a review of the literature. Int J Oral Maxillofac Surg 1990; 19:135-38 CrossRef Medline

6. Marchal F, Dulguerov P, Becker M, et al. Submandibular diagnostic and interventional sialendoscopy: new procedure for ductal disorders. Ann Otol Rhinol Laryngol 2002;111:27-35 CrossRef Medline

7. Brown JE, Drage NA, Escudier MP, et al. Minimally invasive radiologically guided intervention for the treatment of salivary calculi. Cardiovasc Intervent Radiol 2002;25:352-55 CrossRef Medline

8. Bialek EJ, Jakubowski W, Zajkowski P, et al. US of the major salivary glands: anatomy and spatial relationships, pathologic conditions, and pitfalls. Radiographics 2006;26:745-63 CrossRef Medline

9. Yousem DM, Kraut MA, Chalian AA. Major salivary gland imaging. Radiology 2000;216:19-29 CrossRef Medline

10. Drage NA, Brown JE, Escudier MP, et al. Interventional radiology in the removal of salivary calculi. Radiology 2000;214:139-42 CrossRef Medline

11. Guideline summary: ACR Appropriateness Criteria neck mass/adenopathy. National Guideline Clearinghouse (NGC) [Web site]. Rockville, Maryland: Agency for Healthcare Research and Quality; 2012. Available: https://www.guideline.gov. Accessed July 30, 2017

12. Casselman JW, Mancuso AA. Major salivary gland masses: comparison of MR imaging and CT. Radiology 1987;165:183-89 CrossRef Medline

13. Escudier MP. The current status and possible future for lithotripsy of salivary calculi. Atlas Oral Maxillofac Surg Clin North Am 1998;6: 117-32 Medline

14. Harrison JD. Causes, natural history, and incidence of salivary stones and obstructions. Otolaryngol Clin North Am 2009;42:927-47 CrossRef Medline

15. Bodner L. Salivary gland calculi: diagnostic imaging and surgical management. Compendium 1993;14:572, 574-76, 578 passim; quiz 586 Medline

16. Papadaki ME, McCain JP, Kim K, et al. Interventional sialoendoscopy: early clinical results. J Oral Maxillofac Surg 2008;66:954-62 CrossRef Medline

17. Williams MF. Sialolithiasis. Otolaryngol Clin North Am 1999;32: 819-34 CrossRef Medline

18. Schrøder SA, Andersson M, Wohlfahrt J, et al. Incidence of sialolithiasis in Denmark: a nationwide population-based register study. Eur Arch Otorhinolaryngol 2017;274:1975-81 CrossRef Medline

19. Becker M, Marchal F, Becker CD, et al. Sialolithiasis and salivary ductal stenosis: diagnostic accuracy of MR sialography with a threedimensional extended-phase conjugate-symmetry rapid spin-echo sequence. Radiology 2000;217:347-58 CrossRef Medline

20. Marchal F, Dulguerov P. Sialolithiasis management: the state of the art. Arch Otolaryngol Head Neck Surg 2003;129:951-56 CrossRef Medline

AJNR Am J Neuroradiol 38:2161-66 Nov 2017 www.ajnr.org 
21. Rauch S, Gorlin RJ. Disease of the salivary glands. In: Gorlin RJ, Goldmann HM, eds. Thomas' Oral Pathology. St. Louis: Mosby-Year Book; 1970:997-1003

22. Jäger L, Menauer F, Holzknecht N, et al. Sialolithiasis: MR sialography of the submandibular duct-an alternative to conventional sialography and US? Radiology 2000;216:665-71 CrossRef Medline

23. Rzymska-Grala I, Stopa Z, Grala B, et al. Salivary gland calculi: contemporary methods of imaging. Pol J Radiol 2010;75:25-37 Medline

24. Aran S, Daftari Besheli L, Karcaaltincaba M, et al. Applications of dual-energy CT in emergency radiology. AJR Am J Roentgenol 2014; 202:W314-24 CrossRef Medline

25. Johnson TR. Dual-energy CT: general principles. AJR Am J Roentgenol 2012;199(5 suppl):S3-8 CrossRef Medline

26. Barrett JF, Keat N. Artifacts in CT: recognition and avoidance. $R a-$ diographics 2004;24:1679-91 CrossRef Medline

27. Boas FE, Fleischmann D. Evaluation of two iterative techniques for reducing metal artifacts in computed tomography. Radiology 2011; 259:894-902 CrossRef Medline 